\section{Regards sur l'économie allemande}

Bulletin économique du CIRAC

$96 \mid 2010$

Varia

\title{
Priorité de l'Allemagne : concilier vies familiale et professionnelle
}

\section{Werner Eichhorst et Eric Thode}

Traducteur : Isabelle Bourgeois

\section{OpenEdition \\ Journals}

Édition électronique

URL : http://journals.openedition.org/rea/4079

DOI : $10.4000 /$ rea. 4079

ISBN : 978-2-8218-0888-1

ISSN : 1965-0787

Éditeur

CIRAC

Édition imprimée

Date de publication : 1 mai 2010

Pagination : 13-21

ISSN : 1156-8992

\section{Référence électronique}

Werner Eichhorst et Eric Thode, "Priorité de l'Allemagne : concilier vies familiale et professionnelle », Regards sur l'économie allemande [En ligne], 96 | mai 2010, mis en ligne le 01 mai 2012, consulté le 01 mai 2019. URL : http://journals.openedition.org/rea/4079 ; DOI : 10.4000/rea.4079 


\title{
Priorité de 1'Allemagne : concilier vies familiale et professionnelle
}

\author{
Werner Eichhorst, Eric Thode
}

Longtemps, la politique familiale a fait figure de parent pauvre en Allemagne ; et elle ne visait ni la natalité ni l'emploi des femmes. Dans la RFA d'avant l'Unité, et jusque vers la fin des années 1990, son objectif premier était le soutien matériel apporté aux couples mariés avec enfants, plus particulièrement pour amortir les phases de non-activité des mères. Cette approche reflétait le modèle longtemps prédominant d'un père de famille disposant d'un emploi stable et d'un revenu suffisant pour nourrir seul sa famille; le rôle de l'épouse étant de l'éducation des enfants et la gestion du foyer. La politique familiale se traduisait par des mesures de politique fiscale et sociale : imposition jointe des conjoints avec fractionnement, choix entre les catégories d'IR selon les revenus des conjoints, allocations familiales, couverture sociale du conjoint non salarié. Les conjoints les mères, en règle générale - désireux de prendre un emploi pour compléter le revenu familial en étaient plutôt dissuadés par le peu de structures d'accueil de la petite enfance, un régime fiscal défavorable pour le deuxième salaire et par de faibles revenus de substitution versés pour des congés parentaux de longue durée. Longtemps en effet, on estimait que c'est aux mères que revenait en priorité l'éducation des enfants et qu'elles devaient pour ce faire abandonner ou réduire leur activité ; pour seule compensation, les années d'éducation étaient prises en compte par l'assurance retraite, ou les droits à pension de retraite transférés en cas de divorce. Cette politique a eu pour effet de réduire considérablement l'activité professionnelle des mères, ne leur laissant guère que la possibilité d'exercer un emploi à temps partiel, ce qui, aujourd'hui encore, contribue à brider les perspectives professionnelles des femmes.

Depuis dix ans environ, une meilleure conciliation des vies professionnelle et familiale est l'un des objectifs figurant en tête de l'agenda politique allemand, avec cependant une nette priorité pour les familles avec des enfants en bas âge. Un pivot central en est le taux de fécondité particulièrement faible en comparaison internationale : le Rostocker Zentrum zur Erforschung des Demografischen Wandels l'estime à 1,366 enfant par femme en 2008 - soit nettement moins que le taux de reproduction. De nombreuses mesures ont donc été prises au cours des dernières années pour influencer immédiatement ou à moyen terme l'équilibre vie familiale/vie professionnelle : création d'une allocation parentale, développement des structures d'accueil de la petite enfance ou accroissement du nombre d'écoles fonctionnant tout au long de la journée. S'y ajoutent les réformes du marché de l'emploi adoptées dans le cadre des lois Hartz ou la révision du droit relatif aux pensions alimentaires. Reste à savoir si ces réformes et quelques autres encore sont mues par une approche globale et et concluante.

\section{Champs centraux de l'action politique - axes de réformes et incohérences -}

Du fait de la répartition classique des compétences gouvernementales, l'objectif de concilier vies professionnelle et familiale ne peut pas constituer un champ d'action politique propre ; cette action est par définition transversale. Car à la poli- 
1. Transferts publics aux familles

Priorité au soutien matériel à la famille tique familiale au sens étroit s'ajoutent par certains aspects les politiques pour l'emploi, pour l'éducation et la formation, et la politique fiscale ; y jouent aussi un rôle important les systèmes de protection sociale (Gornick/Meyers 2003). Ces dernières années, les pouvoirs publics allemands ont adopté un grand nombre de réformes qui ont, directement ou indirectement, modifié l'équilibre vie familiale/vie professionnelle.

Les transferts monétaires directs (comme les allocations par enfant) et les réductions fiscales à effets indirects (exonérations, ristournes) accroissent le revenu disponible des familles. Ces mesures sont généralement légitimées ainsi: comme les parents contribuent à l'avenir de la société, celle-ci est tenue de leur rembourser dans une large mesure les coûts liés à la famille. Or de telles prestations constituent une incitation économique à fonder une famille. Ainsi, par exemple, une allocation par enfant dont le montant varie selon les revenus peut réduire l'incitation à prendre un emploi ou à étendre l'activité professionnelle, et favoriser de la sorte le partage traditionnel des rôles en ce qui concerne l'éducation et la vie professionnelle. Autrement dit: par leur montant, les transferts publics aux familles influent sur la décision de fonder une famille, par leur architecture, sur la relation entre activité professionnelle et travail familial (pour un aperçu des indicateurs internationaux, voir Eichhorst et al., 2007).

Une comparaison internationale des dépenses publiques consacrées aux familles (selon la méthodologie de l'OCDE) révèle que l'Allemagne figure toujours dans la moyenne haute des pays industrialisés en ce qui concerne le volume global des transferts aux familles, mais que dans le même temps, elle investit relativement peu dans les mesures non pécuniaires. En d'autres termes, priorité reste donnée au soutien matériel accordé à la famille.

Dépenses publiques pour les familles :

transferts, services aux familles et réductions fiscales en \% du PIB en 2005

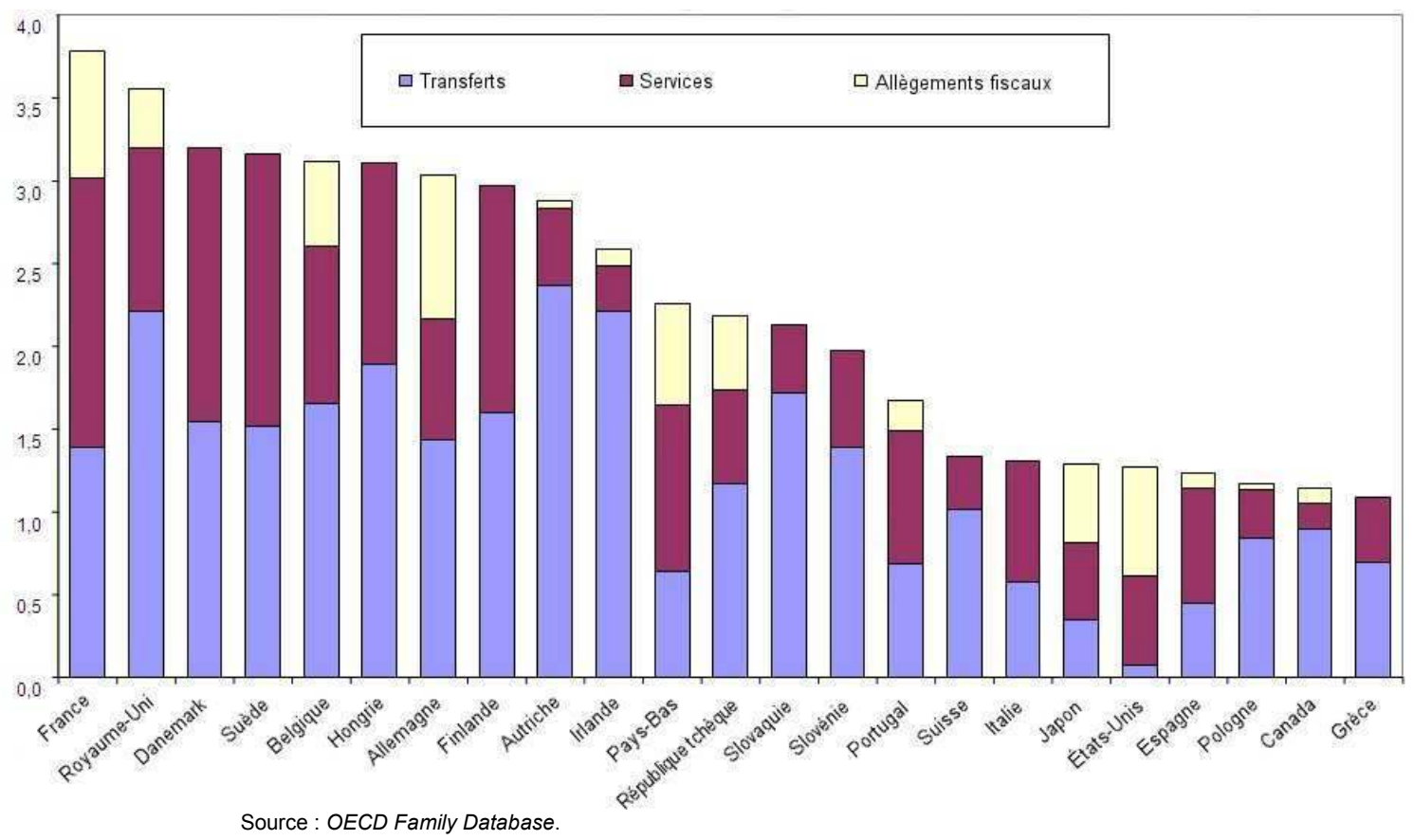

Un soutien pécuniaire généreux
La comparaison internationale révèle par ailleurs que ce soutien (allocations par enfant, franchise fiscale par enfant) est relativement généreux en Allemagne. Avec $184 €$ pour le premier et le deuxième enfant, $190 €$ pour le troisième et $215 €$ pour chacun des suivants, les allocations comptent parmi les plus élevées en Europe, surtout si on prend en considération le fait qu'il s'agit de transferts exonérés d'impôt, contrairement à ce qui est le cas par exemple au Danemark. On notera également que, bien que le montant des allocations soit fonction du 
nombre d'enfants, les familles n'ayant que peu d'enfants bénéficient elles aussi d'un soutien plus fort que dans les autres pays.

Parmi les services de l'Etat aux familles, l'offre de structures publiques d'accueil est de loin la catégorie la plus importante. Cette offre soutient l'activité professionnelle des femmes et des mères et constitue souvent la condition qui leur permet d'occuper un emploi à temps plein ou partiel. Indirectement, elle influence aussi le choix de fonder une famille. Toutefois, l'ampleur des effets directs et indirects de l'offre de structures publiques d'accueil dépend largement de sa forme concrète: qualité des infrastructures, horaires d'ouverture, accueil d'enfants de plusieurs tranches d'âge ou non, mode de financement et coût pour les familles (calculé en fonction du revenu ou non, montant de la contribution parentale), type d'organisme dont dépend la structure d'accueil.

Largement fondée sur les transferts, visant l'allègement des charges pesant sur le salaire principal, et dont le pivot reste le soutien pécuniaire aux familles, la politique familiale allemande a donc toujours pour corollaire une offre limitée d'infrastructures d'accueil en ce qui concerne la quantité (places disponibles) et la flexibilité temporelle (heures d'ouverture), comme le révèle le tableau ci-dessous. Dans l'ouest de l'Allemagne, conformément au modèle du père nourricier, l'offre et la demande de structures publiques d'accueil ont longtemps été faibles; l'éducation des enfants étant à la charge des mères au foyer.

En RDA, la situation était totalement différente : son modèle de société exigeait des femmes qu'elles occupent un emploi. En conséquence, les infrastructures y étaient nettement plus développées, y compris pour les enfants en bas âge, et pour des durées d'accueil longues. Ces infrastructures ont largement persisté dans les nouveaux Länder, et la prestation des services d'accueil des enfants est suffisante dans l'Allemagne de l'Est.

\section{Accueil des enfants}

Une offre traditionnellement faible dans l'ouest de la RFA...

... mais suffisante dans les Länder issus de l'ex-RDA

Dispositif d'accueil des enfants - comparaison internationale, 2007

\begin{tabular}{|c|c|c|c|c|c|c|}
\hline & \multicolumn{2}{|c|}{$\begin{array}{l}\text { Enfants de } \\
\text { moins de } 3 \text { ans }\end{array}$} & \multicolumn{2}{|c|}{$\begin{array}{l}\text { Enfants de l'âge de } 3 \text { ans jusqu'à } \\
\text { l'âge de la scolarité obligatoire }\end{array}$} & \multicolumn{2}{|c|}{$\begin{array}{c}\text { Enfants de l'âge de la scolarité } \\
\text { juqu'à l'âge de } 12 \text { ans }\end{array}$} \\
\hline & $>30 \mathrm{~h}$ & $1-29 h$ & $>30 \mathrm{~h}$ & $1-29 h$ & $>30 \mathrm{~h}$ & $1-29 h$ \\
\hline Danemark & 63 & 7 & 82 & 15 & 65 & 32 \\
\hline Suède & 27 & 20 & 61 & 30 & 100 & 0 \\
\hline Belgique & 23 & 21 & 65 & 35 & 73 & 27 \\
\hline Finlande & 20 & 6 & 55 & 21 & 17 & 83 \\
\hline Espagne & 16 & 24 & 43 & 49 & 50 & 48 \\
\hline France & 15 & 13 & 42 & 51 & 54 & 45 \\
\hline Italie & 15 & 10 & 67 & 23 & 85 & 15 \\
\hline Allemagne & 11 & 7 & 31 & 65 & 36 & 63 \\
\hline Pays-Bas & 4 & 39 & 11 & 80 & 15 & 85 \\
\hline Grande Bretagne & 4 & 34 & 21 & 63 & 64 & 13 \\
\hline Autriche & 1 & 7 & 18 & 52 & 34 & 65 \\
\hline UE 15 & 14 & 20 & 42 & 48 & 55 & 40 \\
\hline UE 25 & 13 & 17 & 41 & 43 & 53 & 41 \\
\hline
\end{tabular}

Source : EU Indicators for Monitoring the Employment Guidelines, 2009 Compendium. NB : Données 2006 pour les enfants de moins de 3 ans en Allemagne.

Dans les Länder de l'ouest en revanche, l'accès fiable à une structure d'accueil, surtout pour les enfants en bas âge (jusqu'à 3 ans), comme la durée de l'accueil (toute la journée, toute l'année) ne répondent toujours pas aux besoins. Quant aux structures d'accueil pour les enfants en âge d'aller à l'école primaire, elles n'étaient longtemps que rarement ouvertes au-delà des horaires de l'école, c'està-dire au-delà de la matinée. Mais les rares places disponibles sont moins chères que dans nombre d'autres pays, du fait que ces structures bénéficient de subventions publiques.
A l'ouest, difficultés d'accès à une structure d'accueil et horaires trop limités 
La hausse de l'emploi féminin incite les pouvoirs politiques à développer les infrastructures

3. Services aux familles. Les sortir du marché noir

4. Congé parental et allocations parentales
Coût de l'accueil des enfants pour les familles

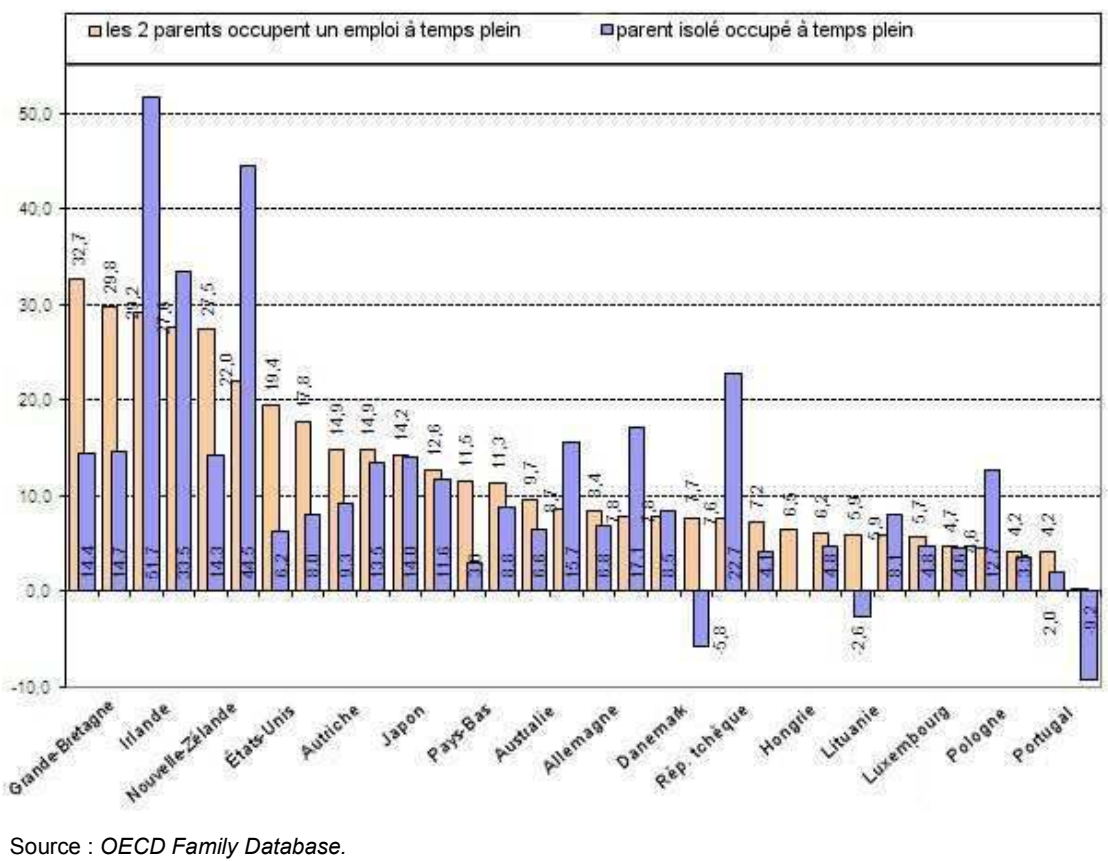

La tendance à la hausse des qualifications des femmes et leur désir en conséquence plus prononcé d'occuper un emploi autre qu'un mini-job ou un temps partiel accroissent le besoin de structures d'accueil et plus particulièrement de structures accueillant les enfants au-delà de la traditionnelle demi-journée. Cette demande se trouve accrue en outre par la réduction de la durée de versement des allocations parentales. Mais depuis la fin des années 1990, les gouvernements SPD/Verts, puis la Grande coalition, ont multiplié les efforts pour remédier à ce déficit d'action politique en développant les infrastructures d'accueil dans les Länder de l'ouest.

En adéquation avec la priorité accordée à l'éducation des enfants par la famille et avec une activité professionnelle des mères réduite à un temps partiel limité, l'offre comme la demande de prestations de soutien aux familles a longtemps été considérée comme secondaire en Allemagne. Or l'éducation au sein de la famille ne permet pas d'acquérir des revenus susceptibles d'entretenir une demande de prestations sur le marché. S'y ajoute, dans le cas de l'Allemagne, le coût relativement élevé des services à la personne du fait d'un fort taux de prélèvements sociaux. La conséquence en est que les services aux familles et aux personnes relèvent aujourd'hui pour l'essentiel de l'économie souterraine. Les pouvoirs publics ont toutefois adopté ces dernières années diverses mesures pour développer le potentiel en termes d'emplois de ces prestations. Ainsi, les prestations aux familles sont déductibles de l'IR à hauteur de $20 \%$ d'un plafond de $20000 €$ par an, soit $4000 €$. S'y ajoute la procédure de déclaration simplifiée des minijobs grâce aux chèques emploi-service. L'Allemagne s'est donc dotée d'un modèle visant à rendre plus accessibles pour les familles les prestations dans le domaine légal.

Les dispositions permettant aux parents de quitter totalement ou en partie, pour un certain temps, la vie active grâce à un soutien pécuniaire pour se consacrer plus à la famille constituent, bien sûr, une incitation à fonder une famille. Mais la réalisation de ce projet dépend largement de la nature concrète de ces dispositions, c'est-à-dire essentiellement de la durée de l'inactivité, du montant comme de la nature du revenu de substitution, de la flexibilité du recours à ces aides, ainsi que de la certitude de pouvoir reprendre une activité appropriée à l'issue du congé parental. Or au fur et à mesure que se prolonge la période d'inactivité, les qualifications comme les savoir-faire sont frappés d'obsolescence, ce qui rend 
plus difficile aux mères ou aux pères le retour en activité. Le risque est donc grand de les voir finalement renoncer à la reprise, pourtant désirée, d'un emploi.

A l'inverse de l'extension graduelle de l'éventail des services aux familles qui se contente au fond d'adapter en le pérennisant le système existant, l'Allemagne a mené une réforme fondamentale avec la création de la nouvelle allocation parentale. Contrairement à l'allocation pour l'éducation d'un enfant (Erziehungsgeld) qui n'était versée qu'aux revenus inférieurs à un certain plafond et était limitée à une période de 2 à 3 ans, l'allocation parentale (Elterngeld) est un revenu de substitution de courte durée, conçu sur le modèle des allocations chômage (deux tiers du salaire net), mais avec une composante de redistribution, puisque les foyers à faibles revenus bénéficient d'un revenu de substitution proportionnellement plus important et que les inactifs perçoivent une allocation minimum de $300 €$ par mois. L'allocation parentale est versée à l'un des parents pendant 12 mois au maximum ; l'autre pouvant bénéficier de deux mois supplémentaires, soit de 14 au total.

Un revenu de transfert modulé en fonction du salaire correspond certes au principe traditionnel et fondamental de l'Etat Providence de type bismarckien, à savoir qu'il compense la perte de revenu salarié pour préserver le statut social du récipiendaire. Mais dans le cas de l'allocation parentale, il s'agit d'une innovation institutionnelle inspirée des modèles danois et suédois qui tendent à relativiser plutôt qu'à pérenniser le principe du père nourricier. En d'autres termes: l'abaissement de la durée du versement vise à inciter les bénéficiaires à réduire la durée d'inactivité et à retourner plus vite dans la vie professionnelle; la modulation de l'allocation en fonction du salaire incite, bien plus que l'ancienne allocation forfaitaire d'éducation, les salariés occupant un emploi à temps plein donc le plus souvent les pères - à prendre un congé parental, incitation accrue par l'octroi des deux 'mois paternels'.

En comparaison avec l'ancienne allocation d'éducation, l'allocation parentale réduit la baisse des revenus pour de nombreux salariés à temps plein et familles aux revenus supérieurs, ce qui contribue pour ce groupe à rendre moins conflictuel le choix entre vie familiale et vie professionnelle. Les statistiques du ministère fédéral de la Famille (BMFSFJ) montrent que, depuis l'instauration de l'allocation parentale, les pères y recourent plus volontiers qu'avant : ils sont $16 \%$ en 2008 , alors qu'ils n'étaient encore que $3,5 \%$ en 2006 . On observe peu de changement en ce qui concerne les mères : elles interrompent toujours leur vie professionnelle plus longtemps que les pères.

Le choix de fonder une famille et de concilier vies familiale et professionnelle est influencé d'une manière générale par le niveau de redistribution au bénéfice des familles qu'institue le régime de l'impôt sur le revenu, tout plus particulièrement via les différentes catégories d'IR. Le point crucial est la question de savoir si, et si oui dans quelle mesure, le régime de I'IR applicable aux membres de la famille occupant un emploi prend en compte les charges correspondant à l'entretien de ses membres inactifs. Le régime de l'imposition individuelle n'en tient pas compte, I'IR étant alors prélevé indépendamment de la situation familiale de chaque contribuable. A l'opposé, l'imposition conjointe a pour référence le revenu de la famille et prend en considération l'apport respectif de ses membres. Dans ce régime, les personnes sans ou à faible revenus que compte la famille ont un droit à l'entretien par celles dont le revenu salarié est plus important. Ce droit réduit le revenu imposable de la famille et lui garantit ainsi un revenu disponible supérieur.

En Allemagne, l'imposition conjointe se pratique sous la forme de l'imposition jointe des conjoints avec fractionnement (Ehegattensplitting). Elle favorise fiscalement le couple marié, quel que soit le nombre d'enfants. L'avantage fiscal annuel est d'autant plus élevé que l'écart entre les revenus des conjoints est grand. Ce régime qui favorise la polarisation de la répartition des revenus a pour premier effet de compenser la perte de revenu des familles où l'un des conjoints se consacre majoritairement à l'éducation des enfants et n'a donc qu'un revenu limité.
Allocation parentale : un changement de paradigme...

... d'inspiration scandinave...

... qui incite de plus en plus de pères à rester au foyer

5. Impôts, cotisations sociales et transferts

Un régime de I'IR pro-famille, mais qui profite aussi aux couples mariés sans enfants 
6. Activité dite négligeable (mini-jobs)

Protection sociale : complémentarité de la couverture selon les liens familiaux

Les mini-jobs aussi sont l'expression du modèle du père nourricier

7. Forfait de base versé aux chômeurs de longue durée

Bénéficiaires de Hartz IV et conjoints divorcés doivent occuper un emploi
Or cette imposition jointe avec fractionnement a un revers : ce système public de redistribution bénéficie en effet également aux couples sans enfants. Et elle a des effets désincitatifs au cas où le conjoint au salaire le plus faible souhaite étendre son activité professionnelle ou même simplement entrer dans la vie active.

La progressivité de l'impôt anéantissant une partie des avantages de l'imposition jointe des conjoints avec fractionnement, la prise d'une activité par le deuxième conjoint mène depuis toujours à une forte charge fiscale marginale. Cela est particulièrement net au-delà de la limite de $400 €$, c'est-à-dire d'un mini-job exercé en complément d'une activité salariée ou à titre d'emploi principal. Endessous de ce plafond de $400 €$, le modèle des mini-jobs permet d'exercer une activité exonérée d'impôts et de cotisations fiscales, apportant un complément de revenu bienvenu au couple, d'autant que le conjoint exerçant une telle activité dite négligeable bénéficie d'une couverture sociale puisque le revenu principal du couple est, lui, largement soumis à cotisations.

Dans le cadre du système d'assurance maladie légale, les membres de la famille sont couverts sans qu'ils aient à cotiser ; depuis 2010 cependant, le fait que les cotisations maladie soient déductibles de I'IR réduit certes le poids des prélèvements subis par les actifs, mais diminue dans le même temps l'avantage relatif de la couverture automatique des membres de la famille inactifs. En ce qui concerne l'assurance retraite, l'inactivité du conjoint ne lui ouvre certes pas de droits à une couverture propre, mais du moins à une couverture indirecte sous la forme d'une pension de réversion lorsque le mariage n'est pas dissous. La logique de la protection sociale continue donc de prévoir une complémentarité des mécanismes fondée sur la nature des liens familiaux.

Le seuil de $400 €$ pour une activité négligeable rend à l'inverse inintéressante, pour le conjoint apportant un revenu complémentaire au sein d'un couple marié, toute activité donnant lieu à une rémunération supérieure à ce seuil, puisque celle-ci est soumise à cotisations sociales et au taux plein de l'IR (en règle générale s'applique alors la classe $\mathrm{V}$ ). Les prélèvements sociaux sont néanmoins progressifs jusqu'à un plafond de $800 €$. Parallèlement, comme les mini-jobs ont le privilège d'être libres de tout prélèvement, aux yeux de ceux qui les occupent, ces derniers ont de plus en plus tendance à accepter même des bas salaires horaires. Etant donné que, du fait de la modération salariale, le salaire principal de la famille (emploi régulier) tend à stagner, la demande d'une activité complémentaire (sous la forme d'un mini-job) pour maintenir le niveau de vie familial s'est accrue. Or depuis l'extension du segment des bas salaires opérée voici bientôt dix ans, les mini-jobs sont très prisés des employeurs puisqu'ils leurs permettent de réduire les coûts salariaux et les charges patronales dans les secteurs intensifs en main-d'œuvre. Ainsi, les mini-jobs sont aussi la manifestation du fait que le segment à bas salaires bénéficie de ce que les actifs qui les occupent participent du revenu salarial comme des droits à protection sociale afférents du conjoint apportant le revenu principal de la famille.

La comparaison internationale révèle que le système allemand de prélèvements fiscaux et sociaux est l'un de ceux qui s'orientent le plus sur le modèle classique, conservateur, de la politique familiale qui allie pour l'essentiel un net avantage matériel pour le conjoint alimentant le couple ou la famille et des structures d'incitation défavorables pour le conjoint apportant le salaire d'appoint.

Dans le cadre de la Loi Hartz IV a été créé un minimum vital pour les chômeurs de longue durée : fonction du degré de nécessité du bénéficiaire, il est clairement axé sur la famille, puisqu'il prend pour référence la «communauté de besoin » (Bedarfsgemeinschaft) qui repose sur le devoir d'entretenir conjoint et enfants. Le minimum vital réduit cependant ces devoirs envers les parents et les enfants majeurs vivant hors du foyer familial. Soulignons que le versement du forfait de base implique que les bénéficiaires adultes sont considérés par principe comme disponibles sur le marché de l'emploi dès que leur enfant a atteint l'âge de 3 ans ; la loi prévoit pour eux un accès amélioré ou privilégié aux structures d'accueil. 
Quant au régime général des pensions alimentaires en cas de divorce, il a lui aussi été révisé pour mieux prendre en considération les besoins des enfants : le standard de vie du couple marié n'est plus le seul critère pris en compte lorsqu'il s'agit d'évaluer si la reprise d'un emploi est acceptable pour le conjoint ou pour calculer le montant de la pension alimentaire. On attend donc désormais du conjoint divorcé qu'il occupe une activité.

Différences entre l'emploi des hommes et des femmes (en équivalent temps plein)

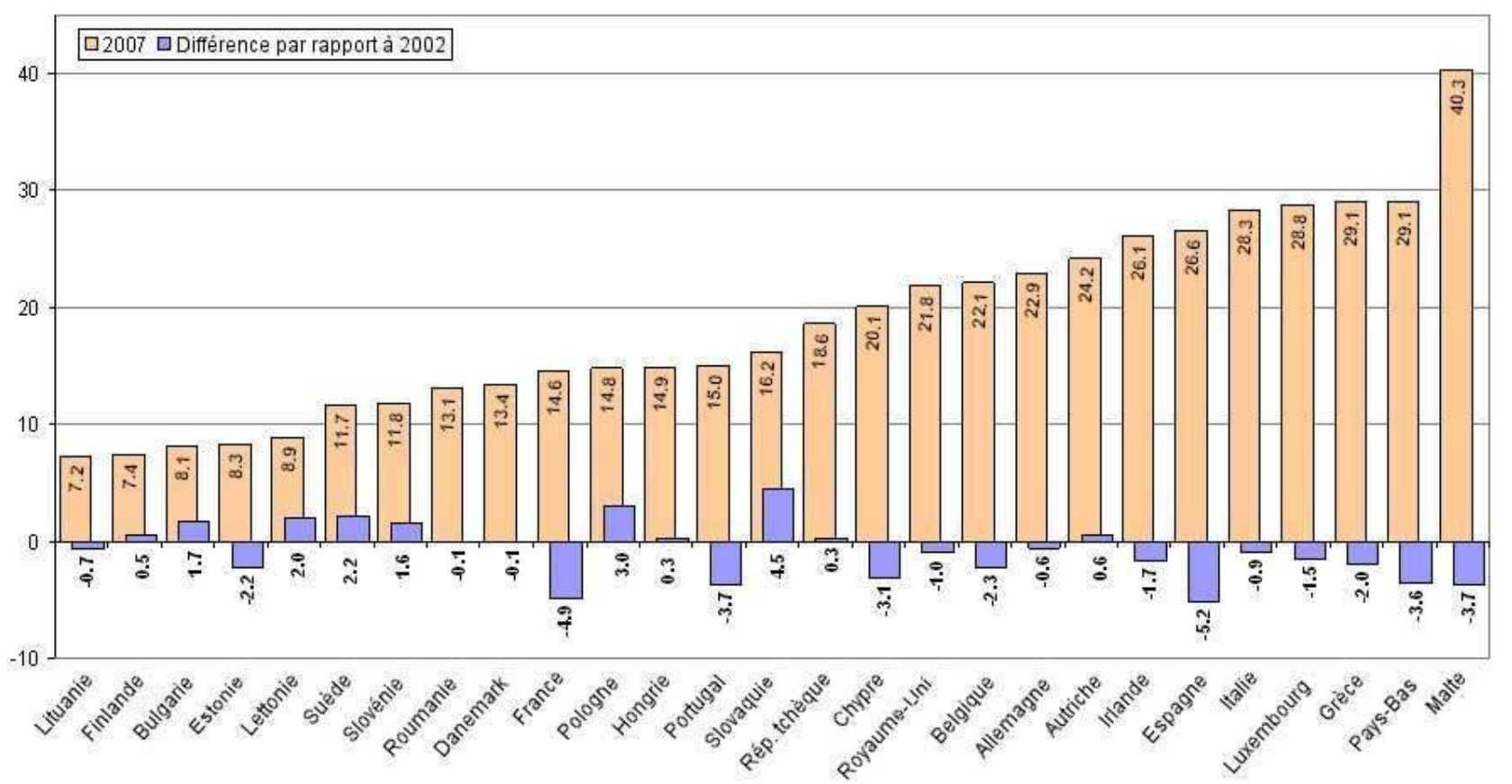

Source : EU Indicators for Monitoring the Employment Guidelines. 2009 Compendium.

Le régime du minimum vital comprend, outre le forfait de base, l'indemnité sociale versée aux enfants ; et il prévoit une indemnité complémentaire pour les foyers avec enfants disposant de faibles revenus, c'est-à-dire dont le revenu permet de couvrir les besoins des conjoints, mais pas des enfants. Actuellement, cela signifie concrètement le versement, illimité par principe, d'une allocation par enfant augmentée de $140 €$ pour tout couple touchant plus de $900 €$ de salaire ou d'allocation chômage ou pour tout parent isolé percevant plus de $600 €$. Au fur et à mesure que les revenus augmentent, l'indemnité par enfant se voit diminuer de moitié. Cet effet « prime pour l'emploi » destinée aux familles à faibles revenus présente d'un côté une forte incitation à garder une activité en plus de la perception du forfait de base, mais réduit de l'autre la propension à sortir du périmètre des revenus de substitution pour prendre un emploi mieux rémunéré ou à temps plein. II en résulte une distinction toujours aussi nette entre l'emploi des hommes et des femmes en équivalent temps plein.

\section{La voie que suivent les réformes en Allemagne}

Loin d'être figés, les Etats providence modernes ne cessent de s'adapter en permanence; par nécessité, ils deviennent donc des modèles hybrides au fil de leur évolution. II importe donc moins de se demander à quel idéaltype ressemble le plus actuellement un Etat providence, que de se demander vers lequel il évolue. Ainsi, en Allemagne, les champs politiques où l'on cherche à concilier vies familiale et professionnelle se caractérisent à la fois par leur invariance et leur changement. D'une part, le pivot du droit de la protection sociale et du code fiscal reste le modèle du père nourricier, assorti des droits à la protection pour le con-
Un effet « prime pour l'emploi » qui pérennise la distinction entre emploi féminin et masculin

Le modèle du père nourricier reste prédominant 
Des changements à la marge qui ne peuvent encore déployer tous leurs effets

Une politique familiale hybride

Un modèle qui reste de type bismarckien joint qui en sont dérivés comme de la prise en considération, au niveau de l'IR, des charges qui en découlent pour subvenir aux besoins du conjoint et des enfants. La faible imposition moyenne des familles a pour corollaire un niveau élevé de prélèvements marginaux frappant les revenus complémentaires du conjoint, dès lors que ceux-ci dépassent le plafond des activités négligeables. En Allemagne, le système de prélèvements privilégie donc un modèle familial où un conjoint pourvoit seul aux besoins du ménage ou bien où se constate une nette polarisation de l'activité et des revenus issus de l'activité : l'un des conjoints travaille à temps plein et rapporte l'essentiel des revenus du ménage, pendant que l'autre exerce un emploi d'appoint.

Alors que ces éléments n'ont guère varié, la loi de réforme instituant le revenu minimum d'insertion pour les chômeurs de longue durée (Hartz IV) a déplacé légèrement l'accent sur les principes de l'activation des chômeurs et de leur capacité à assurer leur propre subsistance. Comme dans le cas de l'indemnité par enfant, on observe une nouvelle dynamique entre la protection matérielle d'un côté et, de l'autre, des mesures d'incitation plus prononcées à la reprise d'un emploi et à l'ascension sociale. La rareté des prestations d'accueil de l'enfance inhibe certes encore une activation plus poussée des bénéficiaires de revenus de transfert comme une meilleure intégration dans l'emploi des parents isolés. La création de l'allocation parentale, en augmentant la demande de structures d'accueil, a accru le problème. Certes, l'allocation parentale est une mesure permettant de réduire les périodes d'interruption de l'activité et d'inciter les deux parents, y compris les pères à revenus supérieurs, à se consacrer pleinement à la famille, mais ce modèle trouve ses limites dans la pénurie de capacités d'accueil pour les enfants en bas âge. Toutefois, en rupture avec la tradition (ouest-)allemande de limiter pour l'essentiel à une demi-journée l'accueil dans les jardins d'enfants, de grands efforts ont été entrepris au cours des dernières années pour étendre l'offre de structures d'accueil. II en va de même du soutien fiscal dont bénéficient les prestations aux familles, afin de les 'légaliser' en les sortant de l'économie souterraine.

On voit dès lors s'esquisser les éléments clefs du nouveau cap poursuivi par la politique familiale allemande :

- caractère invariant des fondements du modèle du père nourricier dans le droit fiscal et social (imposition jointe des conjoints avec fractionnement, couverture sociale automatique du conjoint) ;

- extension limitée du modèle du père nourricier vers celui d'un conjoint augmentant jusqu'à 1,25 à 1,5 fois le revenu de la famille grâce à des mesures d'incitation forte à la prise d'un emploi à temps partiel (essentiellement sous la forme de mini-jobs ou via l'allocation par enfant);

- par-delà, mesures visant à faciliter l'activité des mères grâce à l'extension progressive à l'après-midi des horaires d'accueil pour les enfants (Länder de l'ouest), à l'extension des structures d'accueil de la petite enfance, à l'allongement des rythmes scolaires (matin et après-midi), et grâce aux mesures fiscales en faveur des services aux familles.

Le cap poursuivi par les réformes allemandes se présente comme un mélange complexe, parfois contradictoire, d'approches politiques et institutionnelles aux origines multiples :

- modèle bismarckien, de type continental et conservateur, d'une protection sociale garantissant le statut des assurés : il est au fondement de la couverture maladie automatique du conjoint et des enfants, ainsi que des pensions de réversion au conjoint survivant comme aux orphelins. Viennent compléter cette approche : le dégrèvement fiscal dont bénéficie le salaire principal dans le cadre de l'imposition jointe des conjoints avec fractionnement, le versement d'allocations par enfant généreuses en comparaison internationale, comme le traitement particulier du revenu d'appoint du conjoint occupant un emploi à temps partiel, en ce qui concerne l'IR et les cotisations sociales ;

- éléments d'une politique libérale. Ils imprègnent le principe de l'activation des bénéficiaires de transferts sociaux, de même que le caractère incitatif dévolu aux mini-jobs et à l'indemnité par enfant dans le sens d'une subvention salariale des bas salaires (modèle du Kombilohn), ou encore le soutien fiscal accordé aux services à la famille offerts sur le marché ; 
- éléments du modèle scandinave : s'ils interviennent eux aussi dans la politique d'activation des chômeurs, on les trouve surtout dans l'extension en cours des infrastructures publiques d'accueil de l'enfance, ainsi que dans la nouvelle allocation parentale, cette prestation modulée selon le revenu qui, tout en s'inspirant du modèle scandinave, reste fidèle au modèle bismarckien.

LA POLITIQUE QUE MÈNE L'ALLEMAGNE POUR CONCILIER vies familiale et professionnelle en créant des conditions-cadre favorables en matière de régime fiscal et de transferts sociaux, et en proposant aux familles des services publics ou subventionnés par l'Etat, se présente comme un mélange complexe et parfois contradictoire d'approches. Les domaines traditionnels d'action politique en faveur de la famille que sont la fiscalité ou la protection sociale jouxtent des champs où s'accumulent les réformes, qu'il s'agisse d'accueil des enfants, d'allocations parentales ou de l'activation jusque des parents d'enfants en bas âge. L'Allemagne n'a pas (encore) abandonné son modèle traditionnel de la famille, mais a procédé à une série de modifications institutionnelles venant en complément de celui-ci. La discussion en cours sur l'individualisation de l'IR ou, à l'inverse, sur l'imposition du ménage est directement liée à la question du périmètre de la communauté de besoins. La situation familiale détermine en effet d'une part le revenu imposable, d'autre part, le niveau des prestations auxquelles elle ouvre droit. Or à aucun de ces niveaux, on ne décèle pour l'instant une nette tendance à l'individualisation dans la politique allemande - sauf dans un seul domaine : le passage de l'allocation éducation à l'allocation parentale.

On peut s'attendre, dans un proche avenir, à la poursuite des réformes graduelles surtout en ce qui concerne l'extension des structures d'accueil en reflet de la hausse continue de l'activité des mères. On voit s'esquisser aussi, à moyen terme, une plus large remise en question, voire une réduction des dispositions favorables aux familles dans le code fiscal et le droit de la protection sociale ; on en trouve les prémisses dans l'exonération fiscale des cotisations à l'assurance maladie, ainsi que dans le modèle sur lequel est basé le Fonds pour la santé. De même, dans le cadre des réformes des retraites, de l'instauration du revenu minimum d'insertion par la Loi Hartz IV, ainsi que de la refonte du droit des pensions alimentaires, l'importance accordée aux transferts garantissant le statut social a diminué, alors que, dans le même temps, les femmes tendent à privilégier de plus en plus l'activité professionnelle. Dans l'ensemble, donc, les principes réglementaires et institutionnels les plus divers continueront de coexister. Si cela reflète la continuité du modèle traditionnel dans laquelle s'inscrivent les dispositions existantes, cela révèle aussi le poids croissant des divergences d'approche politiques, comme le montre l'actuel débat sur l'introduction d'un salaire éducatif pour le conjoint choisissant l'inactivité pour élever son ou ses enfant(s).

Traduction : I. Bourgeois

\section{Indications bibliographiques}

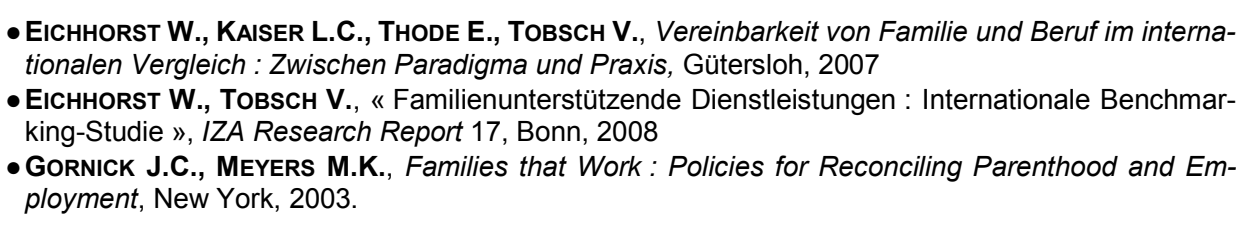

